\title{
Visualizing Cardiovascular Magnetic Resonance (CMR) Imagery: Challenges and Opportunities
}

\author{
Simon Walton ${ }^{\mathrm{a}, 1, *}$, Kai Berger ${ }^{\mathrm{b}}$, Jeyarajan Thiyagalingam ${ }^{\mathrm{a}}$, Brian Duffy ${ }^{\mathrm{a}}$, Hui Fang ${ }^{\mathrm{a}}$, \\ Cameron Holloway ${ }^{\mathrm{c}}$, Anne E. Trefethen ${ }^{\mathrm{a}}$, Min Chen ${ }^{\mathrm{a}, 1, * *}$ \\ ${ }^{a}$ Oxford e-Research Centre, Oxford University, 7 Keble Road, Oxford, OX1 3QG, UK \\ ${ }^{b}$ INRIA Bretagne-Atlantique, Campus universitaire de Beaulieu, 35042 Rennes Cedex, France \\ ${ }^{c}$ St Vincent's Hospital, 390 Victoria St, Darlinghurst NSW 2010, Australia
}

\begin{abstract}
Cardiovascular magnetic resonance (CMR) imaging is an essential technique for measuring regional myocardial function. However, it is a time-consuming and cognitively demanding task to interpret, identify and compare various motion characteristics based on watching CMR imagery. In this work, we focus on the problems of visualising imagery resulting from $2 \mathrm{D}$ myocardial tagging in CMR. In particular we provide an overview of the current state of the art of relevant visualization techniques, and a discussion on why the problem is difficult from a perceptual perspective. Finally, we introduce a proof-of-concept multilayered visualization user interface for visualizing CMR data using multiple derived attributes encoded into multivariate glyphs. An initial evaluation of the system by clinicians suggested a great potential for this visualisation technology to become a clinical practice in the future.
\end{abstract}

Keywords: CMR, visualization, multifield, video, glyph, spatiotemporal

\section{Introduction}

The first observation of atomic nuclei absorbing and emitting radio waves was made by Professor Isidor I. Rabi in 1937, and inspired later work on Magnetic Resonance Imaging (MRI) in the 1970's independently by a number of scientists in the effort to develop a noninvasive scanning method that did not rely on ionising radiation (as does CT scanning). Cardiovascular Magnetic Resonance (CMR) was developed in the 1970's as a specific case of MRI, as an imaging modality 'for clinical studies of the heart and vasculature, offering detailed images of both structure and function with high temporal resolution' [50]. It is sometimes referred to as the 'one-stop-shop for cardiac imaging' [48] as it enables clinicians to assess a comprehensive set of conditions,

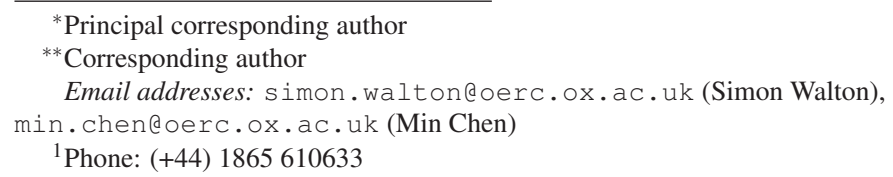


including 'ventricular function, cardiac morphology, vasculature, perfusion, viability, and metabolism' [47]. The demands of a high temporal resolution combined with the rapid movement of the heart resulted in the introduction of introduction of ECG gating in the late 1990's, using the ECG signal of the heart to average many heartbeats together by aligning signal peaks through time [50]. CMR has become an important tool for the assessment of cardiac morphology, function, tissue characterisation, metabolism and perfusion in clinical cardiology.

In clinical and research environments, existing software systems provide users with essential tools for browsing scanned CMR data. For example, physicians can select a particular axial plane, observing the change in scalar values frame-by-frame or as a video. The availability of myocardial tissue tagging helps physicians observe the deformations of the myocardium, whilst facilitating computational processes for obtaining a variety of measurements, such as strain and motion paths. Such data is commonly visualized as a heatmap for scalar values, or an animated vector field for motion and strain direction. It is highly desirable to observe these 'derived' spatiotemporal measurements in conjunction with the original scalar imagery sequence. Because CMR imagery captures 3D volumetric information of a patient's heart over a time period, with both intensity and velocity measures at each $4 \mathrm{D}$ voxel, it has been envisaged that one day clinicians and patients would be able to observe 3D deformation of a heart or blood flowing through a heart. In comparison with the development of visualization techniques for other modalities such as computed tomography (CT), magnetic resonance imaging (MRI) and ultrasound, the research effort made for visualizing CMR data seems to be rather sparse according to the literature.

It is most likely that the lack of technical advancement in visualizing CMR data is due to the 'curse of the temporal dimension'. Nevertheless, there has been a huge amount of research interest in visualizing spatiotemporal data, including flow visualization, time-varying volume visualization and video visualization. One cannot help wonder if any advances in these areas can be transformed to advanced techniques for visualizing CMR imaging data. In this paper, we juxtapose the problems of visualizing CMR data with the relevant advances in visualization. In Section 2, we first examine the state of the art of several areas in visualization, focusing on advanced techniques for visualizing spatiotemporal data. In Section 3, we outline several major challenges in providing appropriate visualization techniques to support clinicians and medical scientists and outline opportunities in research and development. In Section 4, we describe a case study on visualizing multi-layered information derived from cine and tagged image sequences. We offer our concluding remarks in Section 5.

\section{The State of the Art in Imagery Data Visualization}

In this section, we give an overview of the state of the art in imagery data visualization. We begin with a review of recent works on CMR data visualization. We then focus on two subareas of visualization, namely, time varying visualization and video visualization, where the source data exhibits characteristics similar to CMR data. We close this section with a brief overview of other subareas that are relevant to CMR data visualization. 


\subsection{CMR Data Visualization}

The technology of CMR may generate data in the form of a $4 \mathrm{D}$ vector field, a $4 \mathrm{D}$ scalar field, or a subset of such data with reduced dimensionality. Hence a variety of visualization techniques have been applied to CMR data. For example, van Pelt et al. studied visualization of blood flow in a 4D vector field [64, 63]. Volume visualization of 4D CMR data remains a challenge due to the change of intensity of soft tissues in motion. Segmentation of many important structural features, such as endocardial (interior) and epicardial (exterior) borders of a heart, requires a substantial amount of user intervention [48]. Typically visualizations in the CMR literature are streamline representations of blood flow which are superimposed on a greyscale image slice extracted from the corresponding CMR data (e.g., $[17,61])$. Occasionally, a proxy cup or cone shape was used to approximate the surface of a heart [35].

One can often derive new data fields, such as strain, from the raw CMR data. Wünsche et al. [68] used a finite element model to visualize ventricular strain, with color mapping, vector-based displacement, tensor ellipsoids, streamlines, hyperstreamlines, line integral convolution, and isosurfaces. Guttman et al. [21] described a tool that used color mapping for displaying strain and perfusion information. Osman et al. [45] used a combination of summarized pathlines, frame-by-frame color-mapped strain, velocity field hedgehog plots. They depicted radial/circumferential strain rate as bar charts, with groupings indicating the radial area of interest (endocardium, midwall, epicardium). Visualizing strain rate and myocardial thickening using bar charts is common in the literature [11].

\subsection{Time-varying Volume Visualization}

Time-varying volume visualisation has been studied extensively in the literature, though the dominant focus was placed on simulation data. It is a branch of volume visualization, which is a major subarea of visualization and has been applied to almost all 3D modalities in medical imagining. Given a temporal sequence of volumetric scalar fields, the primary goal of visualization is to convey the temporal changes of objects captured in the volumes. Research efforts can be classified into three categories: computational and interaction support, feature visualisation, and visual mapping, Works on computational and interaction support include data structure for efficient computation [55, 65], and functional representation [25], and interactive exploration [2]. Works on feature visualisation include object tracking [56], isosurface and isovolume extraction [58, 69], visualisation of trend relationships [33], and correlation [8]. Works on visual mapping include rendering methods [30,43, 1], temporal coherence [19], context and focus [67, 24], illustration [26, 59], and storyboarding [37].

\subsection{Video Visualization}

Video visualization is a collection of techniques concerned with the creation of a new visual representation from an input video to reveal important features and events in the video. Meaningful information is extracted from videos and conveyed to users in summary or abstract representations. Video visualization assists users in intelligent reasoning by summarizing video content, removing or reducing the burden of viewing videos. Recently, Borgo et al. provided a comprehensive survey on this topic [3]. 
The techniques for video visualization fall into two major categories, namely imagebased techniques and abstract techniques. Image-based techniques focuses on the detection of scenes, shots, frames, segmented objects in videos [46]. These features are then organised into a structural representation of the video, facilitating, video navigation [36, 51, 54, 14], video montage and video synopsis [27] and video skimming $[62,12]$. Abstract techniques focuses on time-varying attributes in a video (e.g., changes in a scene, changes between frames, motion flow and pixel clusters), and summarize these attributes using a small number of visualizations [13, 9].

The development of the subject has been largely influenced by applications in entertainment [20, 44], sports [23, 34], and security [5]. Recently Duffy et al. first applied video visualization to a biomedical application [15].

\subsection{Other Relevant Visualization Techniques}

There are many other visualization techniques that can potentially be deployed in CMR data visualization. As mentioned in Section 2.1, one can derive a variety of measures from CMR data. In order to observe such measures, it is necessary to employ multi-field visualization techniques. The concept was originated from map overlap in geo-spatial data visualization (e.g., $[38,7])$, and the term multi-field encompasses a much broader spectrum, especially in scientific applications. The layered approach has been the primary methodology for visualizing multi-field data (e.g., $[29,31,60])$. In addition, glyph-based visualization has been used to encode multiple attribute dimensions $[66,4]$, and several authors have studied their use in visualizing diffusion tensor imaging data [52, 28]. Maguire et al. used a taxonomy-based visual design methodology for designing a large collection of glyphs [40]. With the rapid advancement of the medical imaging technology, the quality of the 4D vector fields in CMR will improve, and thus the demand for flow visualization will follow. There is a huge collection of flow visualization works in the literature. There are several surveys on the state of the art in different aspects of flow visualization $[49,32,41,16]$.

\section{Motivation, Challenges and Opportunities}

Analysing the function of the heart is important not just for the diagnosis of heart dysfunction/disease, but also for planning treatments and understanding the effect of drugs on heart function. The expertise in identifying a problem is based upon the wealth of knowledge and intuition developed through years of experience of viewing CMR data in its traditional visual representation of animated imagery. It is extremely important therefore to maintain this original context, presenting the data 'as-is'. To assist with diagnosing these conditions exhibiting complex spatiotemporal behaviour, we can additionally augment such a representation by providing a visualization 'toolbox' in which the CMR imagery is augmented with additional information from both the original and derived data (such as strain, motion, etc.). Such a method allows the clinician to remain in full control of the diagnosis, and additionally can assist the process of doctor-patient communication.

Although all the techniques described in Section 2 deal with spatiotemporal data, they do not seem directly applicable to CMR data. There are several technical challenges in developing techniques for visualizing CMR data. 
Dimensionality. Observing a CMR scan requires information to be conveyed at least in two spatial dimensions and one temporal dimension. Because a 2D image typically occupies two spatial dimensions, there is no spatial dimension left on a computer display for the third dimension of the heart volume or the temporal dimension. In a clinical setting, physicians typically build a holistic mental image about the data by inspecting different axial planes (i.e., 2D space) and viewing the corresponding imagery sequences (i.e., 1D time). This incurs a substantial amount of cognitive load in memorizing phenomena noticed in separate observations as well as in integrating different spatiotemporal information together. Such difficulty can be explained by the limited capacity of short-term memory [42], and known phenomena in human vision such as change blindness [57] and inattentional blindness [39]. In addition to the intrinsic spatiotemporal dimensions, there can be more than one attribute dimension, that is, every spatiotemporal point can be associated with multiple attribute values (e.g., density, velocity, strain, voxel classification, and so on). There is often a need to examine different attributes conjointly or comparatively.

Data Quality. By nature, data obtained via an MRI process contains sampling noise. Obtaining velocities from CMR is difficult, partly due to sampling noise, and partly due to the internal smoothness of the myocardium / blood; such low-gradient areas present difficulties for optical flow algorithms. To add to the difficulty, CMR data is often of a poor resolution, and the density of the heart tissue changes periodically. With myocardial tissue tagging, the signals of the virtual grid structure fade gradually as the initial charge is lost. All these result in difficulties in reconstructing a heart model from the imagery data using either segmentation or volume visualization.

Learning Curve. Viewing animated image sequences relating to a particular axial plane is intuitive and convenient. Software for supporting such basic functionality is usually easy to learn and operate. In order for any visualization techniques to deliver added value, they likely involve novel visual representations that would demand extra learning effort.

Task Sensitivity. The objective of visualizing CMR data is highly sensitive to the tasks of clinicians and medical scientists. Different tasks may require observing different parts of the heart, different motion patterns, and different attributes. Some tasks may require a holistic view of the geometric deformation, while others may require a comparison of several landmark features. Some tasks may be performed in conjunction with specialised medical knowledge, which others will support collaborative activities or communication with patients. Hence, it is unlikely that a single visual design can meet the requirements of different tasks.

Meanwhile, these challenges also present us with new opportunities for research and innovation. When we consider the feasibility of visualizing CMR data in detail, it is not difficult to find that many opportunities do exist.

Different Objectives. There are opportunities for developing different visualization techniques to support different objectives of visualization, which may include supporting routine observation and measurement of different medical conditions, providing external memorisation of spatiotemporal patterns, reducing the need for watching animated image sequences repeatedly, enabling researchers to hypothesise and evaluate different causal effects, communicating with patients, and disseminating findings to medical colleagues. 
Specialised Knowledge. Clinicians and medical scientists who work with CMR data possess certain knowledge that may allow effective dimensionality reduction in visual design. For example, if the visual representations for a subset of imagery data (e.g., invariant background, and normal phenomena) can be omitted or simplified through image processing, video visualization or registration techniques for eliminating based on spatial location, more visual bandwidth can be freed to deliver more meaningful and interesting information.

A Variety of Visual Channels. In addition to the captured imagery data, the dominant visualization technique in existing CMR software is color mapping for delineating different regions of an image. While the color heatmap is effective for a single variable, it is usually unhelpful in supporting multivariate visualization. Meanwhile, many visual channels are yet to be fully explored in CMR data visualization. For instance, some 27 classes of visual channels in four main categories (i.e., geometric, optical, topological and semantic) are listed in [10], offering a wide spectrum of design options to new techniques in CMR data visualization.

Advances in Visualization. As discussed in Section 2, many concepts, methods and techniques have already been proposed and experimented in previous works on flow, volume and video visualization. It is advantageous to map out these prior works systematically in the landscape of CMR data visualization. This will not only reduce cost-ineffective effort for reinventing the wheel, but also enable researchers in CMR data visualization to identify best practices as well as technical gaps. In addition, it is beneficial to explore opportunities where visual and analytical techniques are closely coupled together and complement each other.

\section{Case Study on Visualizing Time-varying Multi-fields Derived from CMR Data}

In this case study, we focus on one of the aforementioned challenges, attribute dimensionality, and in particular, address the need to visualize multiple attribute fields derived from axial imagery sequences. Given a CMR imagery sequence, we can compute many additional attributes associated with the data. For example, we can use an optical flow algorithm to extract a motion vector field that provides a per-pixel estimation of the movement for each frame. Another attribute commonly used for research into the LV is circumferential strain [21], for which the output is a set of strain tensors for each pixel at each time step. At each individual time step, the problem of visualizing multiple attributes in the corresponding spatial context is a classical problem of multi-field visualization. When this problem is combined with the requirement to convey temporal dynamics of each attribute field, the problem bears a striking resemblance to video visualization, except it is perhaps more complex.

By focusing on time-varying multi-fields derived from CMR data, we can develop new visualization techniques for supporting user tasks in handling this class of data. The task requirements thereby include (i) viewing multiple attributes conjointly and comparatively, (ii) alleviating the effects of change-blindness and inattentional blindness, (iii) providing external memorisation, (iv) ensuring visual separability of different attribute fields, and $(v)$ supporting both overview and details on demand. To combine these visual representations of different attribute fields, a layering strategy is used that 
combines lower-frequency data at the bottom (i.e. data that is inherently dense / imagebased) with higher-frequency data on top (i.e. derived attributes of the data encoded into glyphs) into one coherent visualisation that can be viewed either temporally or summarised into a single image. Our system integrates repositionable (or pinnable) glyphs into the visualisation which act as probes into the derived attributes.

The coming sections discuss our layer-based visualisation framework in more detail, including the design of the layers, glyphs, and a system implementing these features into a proof-of-concept user interface.

\subsection{Data Modality}

Before continuing, we discuss the modality of the data used in our work. We concentrate on the temporal 2D CMR data most commonly used in clinical practice. A typical set of data from a scanning session comprises:

- Cine Images - This is a sequence of images $\mathbf{I}_{1}, \mathbf{I}_{2}, \ldots, \mathbf{I}_{n c}$ at $U \times V$ resolution for $T$ timesteps, and typically have resolution of around $T \approx 25, U \approx 200, V \approx 120$.

- Tagged Images - This is a sequence of images $\mathbf{I}_{1}, \mathbf{I}_{2}, \ldots, \mathbf{I}_{n t}$ at $U \times V$ resolution for $T$ timesteps, and typically have resolution of around $T \approx 12, U \approx 250, V \approx$ 190. The scalar values represent the standard cine values overlaid with a tagging grid.

These images may be obtained for multiple slices (basal, mid, apex) of the heart. We concentrate on the basal slice through the short-axis plane as the clinically significant twisting motions of the heart are revealed through this combination. Our collection of scans from research patients covers a variety of pathologies, including healthy patients and scans performed before and after surgery.

In addition to this data, additional derived attributes can be computed, which we discuss next.

\subsection{Computing Additional Attributes using Image Processing}

A tagged CMR image sequence allows us to estimate a motion field that can be used to derive other geometric fields. Such fields can be precomputed offline for the full CMR sequence, with the most expensive operation being the strain computations which require around five seconds of processing time for our test data. This can be reduced further with GPU implementations. Once this precomputation has occurred, the glyphs can be moved around in real-time. Our visualisation system utilises a number of image processing techniques that provide computed attributes to the glyphs for display. These techniques are discussed here.

\subsubsection{Motion Vector Field}

The addition of a tagging grid in the imagery allows optical flow techniques to compute the motion for the heart tissues over time as vector field $\mathbf{u}$. The method assumes that an object at $(x, y)$ may move by a small displacement $(u, v)$ between time $t$ and $t+\delta t$, and the intensity of this object is relatively constant during this $\delta t$ and there is a relatively small displacement. In this work, we utilised the optical flow algorithm designed by Brox et al. [6] to track the heart movement in the sequences. 


\subsubsection{Strain Calculation}

In order to analyse the radial and circumferential strain in a short axis animation of the heart, the displacement vector field $\mathbf{u}$ can be transformed to cylindrical coordinates $\mathbf{u}_{c}(r, \theta, z)$ where in the 2D case, $z=0$. This transformation of coordinates allows for analysis of circumferential strain around the ventricle in material coordinates. Since the strain is not uniformly distributed through the myocardium, we define the stress at a particular point using a Lagrangian strain tensor $\varepsilon$ using a cylindrical coordinate system. The tensor matrix $\varepsilon$ results in three Eigenvectors $\vec{v}_{1}, \vec{v}_{2}, \vec{v}_{3}$ pointing in the maximum, medium, and minimum principal directions of stress with associated Eigenvalues $\lambda_{1}, \lambda_{2}, \lambda_{3}$ denoting the scale of the stress. The major and minor principal directions of strain are particularly interesting as they should indicate the directions of maximal stretching and maximal shortening, which in a healthy heart line up in the radial and circumferential directions respectively [22]. Note that the accuracy of estimating strain in two dimensions is affected by the fact that tissue and blood are moving in 3D (and thus partly perpendicular to the image plane) in the original source.

\subsection{3. 'Ghost' Boundary}

Our visualisation introduces the concept of a ghost boundary which displays a 'residual' boundary of the left ventricle's boundary at maximum systole and diastole (see Figure 3(d) shown as a white outline). The concept is similar to the residual peaks that feature in the frequency histogram outputs of hi-fi systems. Our approach to finding this boundary is to combine per frame level set-based curve fitting with a distance field evaluation. The algorithm is initialized with an elliptical estimate of the LV in the sliced image sequence, e.g. by user definition. A scalar distance field representing the minimum distances to the LV boundary from each pixel $D \subseteq \mathbb{R}^{2}$ is defined such that for a location $p(x, y) \in \mathbb{R}^{2}$ :

$$
D(p(x, y))=\left\|\operatorname{diag}(a, b)^{-1} \cdot R\left(-\theta_{\text {major }}\right) \cdot(p(x, y)-z(x, y))^{\perp}\right\|_{2},
$$

with $\theta_{\text {major }} \in[0,2 \pi]$ the rotation angle of the major semi-axis, $a, b \in \mathbb{R}$ the major and minor semi-axis scaling and $z(x, y) \in \mathbb{R}^{2}$ the centre location of the elliptical estimate. The implemented level set evolution can be expressed as:

$$
\frac{\partial C_{t}}{\partial s}=\alpha F_{1}+\beta F_{2}+F_{3}
$$

where $F_{1}=a(x, y) \vec{N}$ is the constant expansion and shrinking field, $F_{2}=b(x, y) \cdot \kappa \cdot \vec{N}$ is the curvature force, and $F_{3}=(\vec{S} \cdot \vec{N}) \vec{N}$ is a force field. $\alpha, \beta$ are constants and $C_{t} \subset \mathbb{R}^{2}$ is the evolved curve, its smoothness is an input parameter to the algorithm. For each frame we then compute the level sets of the image exploiting the inherent feature of a noticeable image gradient along the endocardium. Given these level sets, we define the ghost boundary $G_{t} \subset \mathbb{R}^{2}$ for a frame $t$ to be:

$$
G_{t}=\underset{C_{u}}{\arg \max }\left(\frac{\int_{C_{u}} D\left(r_{u}(s)\right)\left|r_{u}^{\prime}(s)\right| d s}{\int_{C_{u}}\left|r_{u}^{\prime}(s)\right| d s}\right), u=0 \ldots t,
$$



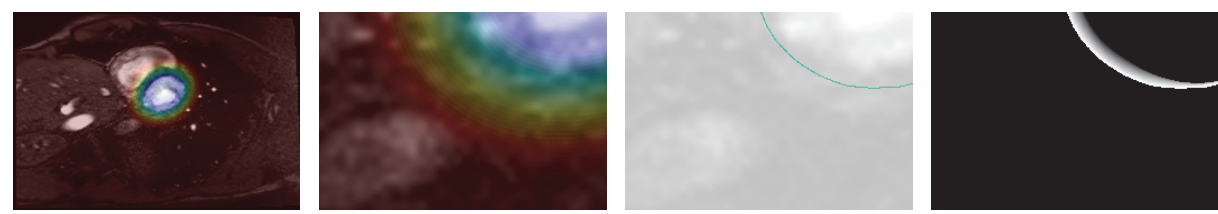

Figure 1: The computation of ghost boundary, the largest circumferential extend of the left ventricle of the CINE loop. Far Left: A frame from the sequence with distance map $D$ overlaid. Middle Left: A close-up near the left atrium. Middle right: level-set curvature weighted with the distance field. Far right: The most current ghost boundary and the history of its evolution from previous ones intensity-coded.

with $r_{u}:\left[s_{0}, s_{n}\right] \rightarrow C_{u}$ an arbitrary bijective parametrization of the curve $C_{u}$. Hence, $G_{t}$ is the contour with the largest distance in $D$ over the cine loop up to frame $t$. An example of $C_{t}$ in $D$ is shown in Fig. 1, middle right. In order to emphasize the "evolution" of $G_{t}$ from a preceding ghost boundary $G_{u<t} \neq G_{t}$, we may interpolate between corresponding positions on the contours:

$$
G_{t, u<t}=\gamma r_{t}\left(s_{i}\right)+(1-\gamma) r_{u}\left(s_{i}\right), i=0 \ldots n,
$$

to arrive at a subset $G_{t, u<t} \subset \mathbb{R}^{2}$ containing $G_{t}$ and $G_{u<t}$ (shown in Figure 1, right with $\gamma$ intensity-coded).

\subsection{Using 'Pinnable' Glyphs}

The use of glyphs for encoding multivariate data is a powerful tool for providing multivariate information in a compact representation, and has been underused in medical visualisation to date [53, 52]. Ward [66] defines a glyph as "a visual representation of a piece of data where the attributes of a graphical entity are dictated by one or more attributes of a data record". Glyphs have been shown to be useful in medical applications due to their ability to provide pre-attentive visualisations to raw imagery derived from scanning sources [53, 52]. Fuchs et al. [18] provide a thorough overview of glyph-based visualisation for the scientific community.

As discussed, augmenting a visualization by providing a toolbox of sorts to the user provides many advantages to the visualization user. Our system provides a set of glyphs that comprise the topmost elements of the visualisation, and are positioned on specific areas of the heart in order to provide measurements from that region. For this reason, our method is not dependent upon image registration between datasets since the user's expertise and knowledge are used in the design and usage of the visualization system.

\subsubsection{Glyph Classes}

As shown in Table 1, after applying various preprocessing techniques, the CMR imagery has been transformed to a multi-field dataset. Although individual fields may be displayed individually using a heatmap in the derived field layer or vector graphics in the primitive layers, glyphs offer much more information capacity if used in a small number of spatial locations on the CMR imagery. By designing a set of glyphs, we can provide clinicians a set of probing tools for investigating some 'information rich' 


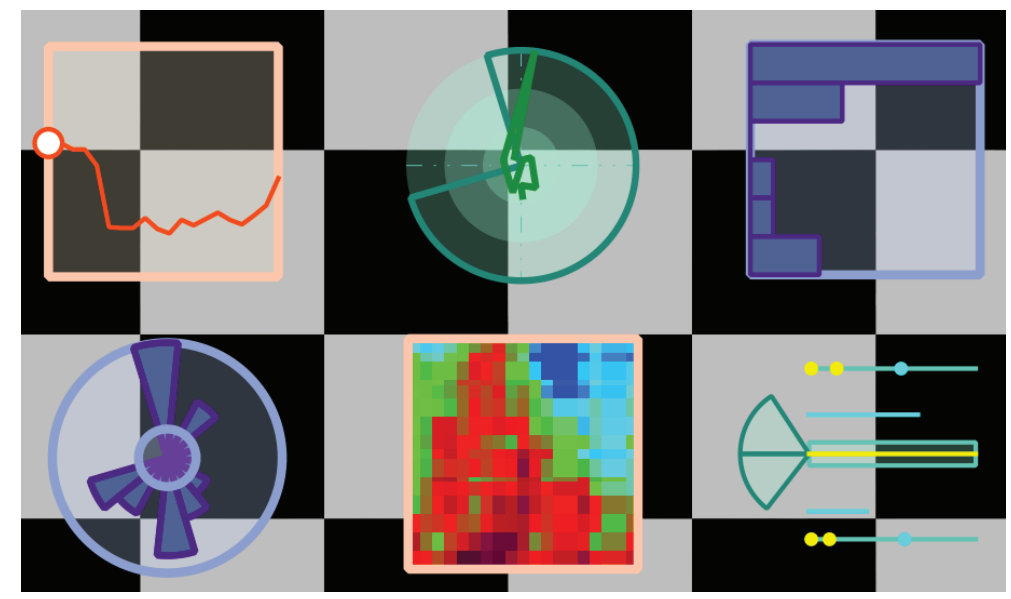

Figure 2: Six example glyphs, top row: scalar time series, vector time series, scalar histogram; bottom row: vector histogram, line profile and geometry summary; green for geometrical, purple for physical and orange for optical attributes. (web \& print)

spots. Making use of a taxonomy-based design pattern [40], we organised our glyphs into classes based on the categorization in Table 1. We designed a metaphorical color scheme to facilitate memorization, i.e., G: geometry $\rightarrow$ green, $\mathrm{P}:$ physical $\rightarrow$ purple and $\mathrm{O}:$ optical $\rightarrow$ orange.

In each major class, we have four standard designs of glyphs for scalar time series, vector time series, scalar histogram, and vector histogram as shown Fig. 2. In addition, we introduce a line profile glyph, which allows users to define a line segment on CMR imagery, and the glyph will display the intensity changes of the line during the video cycle. We also designed a summary geometry glyph for depicting a few key geometrical indicators (i.e., $5 a, 5 b, 5 c, 5 d, 6 a, 6 b$ in Table 1) explicitly or implicitly.

Figure 2 shows examples of our glyphs summarising the temporal properties of the full cycle for their chosen position on the imagery. Moving from the top left along the top row, we have $(i)$ an optical class glyph displaying a scalar timeseries of image intensity; (ii) a geometrical glyph showing cumulative optical flow vectors; and (iii) a physical class glyph showing the image intensity. In the bottom row, we have (iv) another physical class glyph, but this time summarising vector direction and magnitude of optical flow; $(v)$ the specialised line profile glyph showing thickening of the myocardium; and finally (vi) a geometry class glyph summarising key geometrical indicators (see Table 1).

As well as providing a means of bringing desired attributes back into the visualisation on a localised basis, the glyphs also provide the unique function of temporally summarising the data to a user-defined range of the heart cycle (e.g. whole cycle, or systole only). The visualisation now benefits from not only elements that display a single temporal point, but also glyphs that summarise each temporal point in a spatial manner. Summarising temporal data into a single static visual form also has the advantage of reducing perceptual difficulties with temporal data such as change blindness 
Table 1: Different fields and attributes associated with CMR imagery data.

\begin{tabular}{|c|c|c|c|c|}
\hline Field/Attribute & Data Type (per point) & Symbol & Layer & Visual Mapping \\
\hline \multicolumn{5}{|l|}{ Primary Fields } \\
\hline 1. Cine CMR Video & scalar time-series & $S_{\text {cine }}(x, y, t)$ & primary field & video loop \\
\hline 1a. key frame & scalar value & $S_{\text {ckey }}(x, y$, Frm $)$ & primary field & static image \\
\hline 2. Tagged CMR Video & scalar time series & $S_{\text {tag }}(x, y, t)$ & primary field & video loop \\
\hline 2a. key frame & scalar value & $S_{\text {tkey }}(x, y, F r m)$ & primary field & static image \\
\hline 3. Color-mapped CMR Video & time-varying RGB values & $S_{\text {cmap }}^{3}(x, y, t)$ & primary field & video loop \\
\hline 3a. key frame & RGB value & $S_{m k e y}^{3}(x, y, F r m)$ & primary field & static image \\
\hline \multicolumn{5}{|l|}{ Geometrical Attributes } \\
\hline 4. Estimated Motion Flow & vector time series & $V_{m t n}(x, y, t)$ & primitive graphics & animated arrows \\
\hline 4a. magnitude series & scalar time series & $S_{m m t n}(x, y, t)$ & pinnable glyph & glyphs \\
\hline 4b. direction series & $2 \mathrm{~d}$-vector time series & $V_{d m t n}(x, y, t)$ & pinnable glyph & glyphs \\
\hline 4c. average vector & $2 \mathrm{~d}$-vector & $V_{\text {avgmtn }}(x, y)$ & primitive graphics & static arrows \\
\hline 4d. average magnitude & scalar value & $S_{\text {avgmmtn }}(x, y)$ & derived field & heatmap \\
\hline 4e. magnitude range & 3-scalar values & $S_{r m t n}^{3}(x, y)$ & pinnable glyph & glyph component \\
\hline 4f. magnitude histogram & $k$-bin scalar histogram & $H_{m m t n}(x, y)$ & pinnable glyph & glyphs \\
\hline 4g. direction histogram & $k$-bin $2 \mathrm{~d}$-vector histogram & $H_{d m t n}(x, y)$ & pinnable glyph & glyphs \\
\hline 5. Track Path (TP) & array of points & $P_{t p}(x, y)$ & primitive graphics & static polylines \\
\hline 5a. path length & scalar value & $S_{\text {tplen }}(x, y)$ & pinnable glyph & glyph component \\
\hline 5b. curvature & scalar value & $S_{t p c r v}(x, y)$ & pinnable glyph & glyph component \\
\hline 5c. area & scalar value & $S_{\text {tparea }}(x, y)$ & pinnable glyph & glyph component \\
\hline 5d. length/area & ratio value & $S_{t p r l a}(x, y)$ & pinnable glyph & glyph component \\
\hline 6. Straight Line Path (SLP) & displacement vector & $V_{s l p}(x, y)$ & primitive graphics & static arrows \\
\hline 6a. line length & scalar value & $S_{\text {slplen }}(x, y)$ & pinnable glyph & glyph component \\
\hline 6b. SLP-length/TP-length & ratio value & $S_{\text {slprlen }}(x, y)$ & pinnable glyph & glyph component \\
\hline \multicolumn{5}{|l|}{ Physical Attributes } \\
\hline $\begin{array}{l}\text { 7. Strain (Circum./Radial) } \\
7 a-7 g \text { similar to } 4 a-4 g\end{array}$ & vector time series & $V_{\text {strn }}(x, y, t)$ & primitive graphics & animated arrows \\
\hline \multicolumn{5}{|l|}{ Optical Attributes } \\
\hline 8. Intensity Profile & scalar time-series & $S_{\text {cine }}(x, y, t)$ & pinnable glyph & glyphs \\
\hline 8a. line profile & scalar time-series & $S_{l p r f}(x, y, t)$ & pinnable glyph & glyphs \\
\hline 8b. circle profile & scalar time-series & $S_{c p r f}(x, y, t)$ & pinnable glyph & glyphs \\
\hline $\begin{array}{l}\text { 9. Gradient } \\
9 a-9 g \text { similar to } 4 a-4 g\end{array}$ & vector time-series & $V_{g r d t}(x, y, t)$ & primitive graphics & animated arrows \\
\hline 10. Spatial Salience (Variance) & scalar time series & $S_{s s \ln c}(x, y, t)$ & pinnable glyph & glyphs \\
\hline 10a. average spatial salience & scalar value & $S_{\text {avgsslnc }}(x, y)$ & derived field & heatmap \\
\hline 11. Temporal Salience (Variance) & scalar value & $S_{t \operatorname{sln} c}(x, y)$ & derived field & heatmap \\
\hline 12. Gabor Filter & vector time series & $V_{\text {gabor }}(x, y, t)$ & primitive graphics & animated lines \\
\hline 12a. average vector & 2d-vector & $V_{\text {avggabor }}(x, y)$ & primitive graphics & static lines \\
\hline 13. Edge Detector & scalar time-series & $S_{\text {edge }}(x, y, t)$ & pinnable glyph & glyphs \\
\hline 13a. average edge & scalar value & $S_{\text {avgedge }}(x, y)$ & derived field & emulated contours \\
\hline 14. Ghost Boundary & scalar value & $S_{\text {ghost }}(x, y)$ & derived field & emulated contours \\
\hline
\end{tabular}



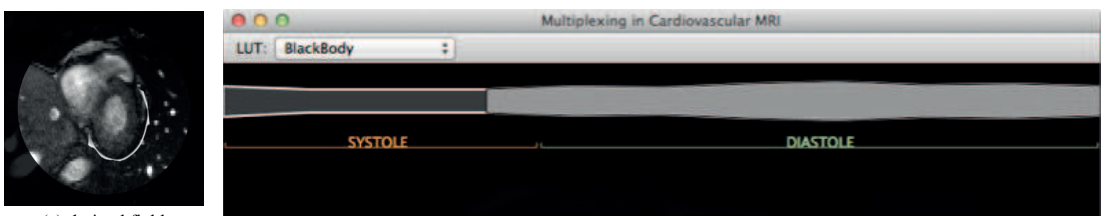

(a) derived field

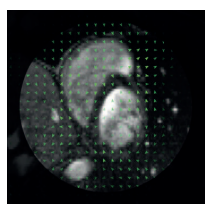

(b) primitive graphics

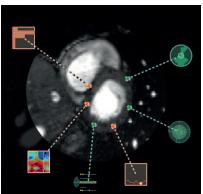

(c) pinnable glyphs

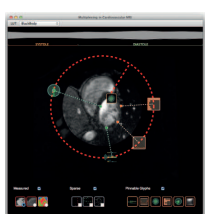

(d) interaction

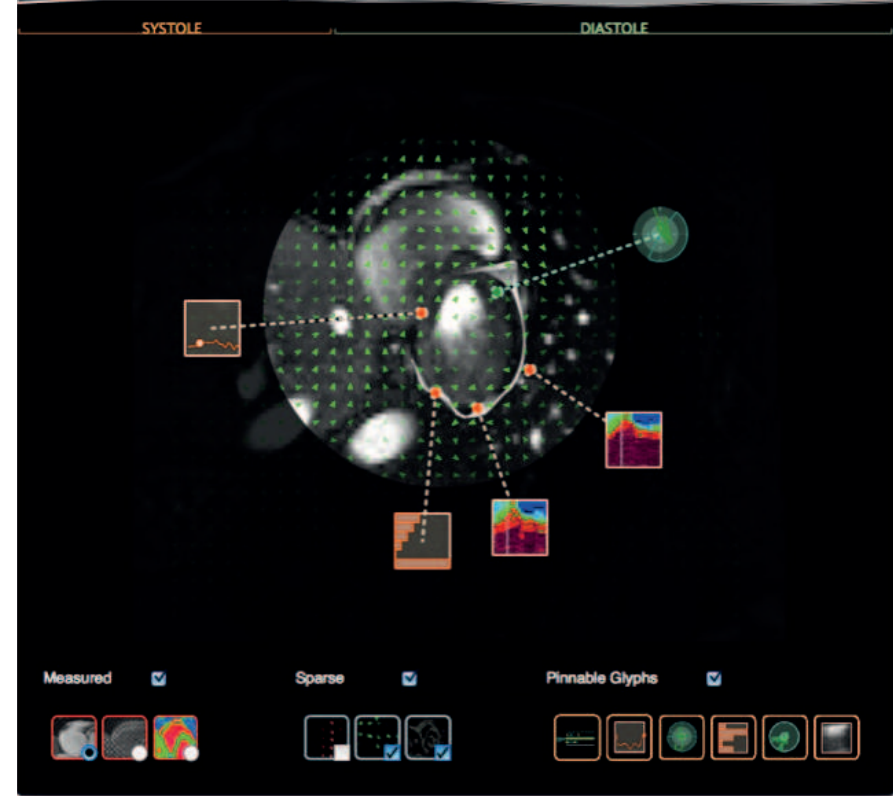

(e) user interface and composition of all 4 layers

Figure 3: Examples of different CMR data layers. (a)-(c) shows how three different layers may be overlaid on top of the primary layer. (d) shows an interaction for placing a pinnable glyph's 'probe' on the heart, with the glyph's 'display' connected outside the circle. (e) shows the user interface and the composition of all four layers. The glyphs shown are (clockwise, from 2 o'clock) (i) the vector timeseries glyph showing summarised strain direction; (ii-iii) two line profile glyphs showing myocardial thickening, (iv) the histogram glyph showing image density; and ( $v$ ) the scalar time series glyph showing image density. (web \& print)

and inattentional blindness (discussed previously).

\subsubsection{Imagery Glyph}

The Imagery glyph summarises attributes of the raw imagery, acting as a thickening indicator which shows the thickening of the myocardium wall at the glyph's circumferential position over time. The glyph's $x$-axis represents time, and its $y$-axis represents a one-dimensional radial slice of the LV running from the computed LV centroid, through the glyph, and out of the outer wall of the LV. By default, the scalar values are derived from the cine imagery and are color-mapped using a standard diagnostic color map used for cine images, although the color map can be changed or disabled entirely.

This glyph serves the additional function of displaying the contraction distance of both walls as the image sampling position is fixed. For clarity, the radial slice axis is always shown along with the glyph, and the time steps are deliberately visually discretised along the $x$-axis to emphasise that the glyph's spatial context is separate 
from the imagery below. To further emphasise the time axis, a small, non-distracting highlight continually animates along the two $x$-axis walls.

\subsubsection{Geometry Glyph}

The Geometry glyph summarises attributes of the motion of the heart due to the motion at the point it is placed, utilising the vector field derived from the motion tracking algorithm. As previously mentioned, the left ventricle's self-intersection flow pattern makes overall flow summarisation difficult; however, the flow of the ventricle at a particular point tends to be slightly asymmetric in nature. The motion at a particular position is represented by sampling the motion vectors in the vector field at the glyph's position over time. If $v f(p, t)$ is a vector field sampling function at point $p$ at time $t$, and $p_{g}$ is the glyph's pinned position, then the vector set is defined as $v f\left(p_{g}, 1\right), v f\left(p_{g}, 2\right), \ldots v f\left(p_{g}, n\right)$. From the resulting vector list, a set of points $p_{1}, p_{2}, \ldots, p_{n}$ are defined by chaining the vectors together into a path, extracting the beginning, end, and intermediate vector link points.

To provide a visual cue as to which parts of the path belong to the systole phase and which parts belong to diastole, the vector path is broken into two sections and colorcoded according to the system's standard systole / diastole colors. From the properties of the vectors, the point in time at which the path breaks between systole and diastole can be obtained from the global vector field statistics. An additional bar to the side of the glyph gives a straightened ratio of the systole to diastole path lengths, which indicates which phase of the heart is producing the most movement at a particular point.

\subsubsection{Physical Glyph}

The Physical glyph summarises the inferred physical properties of the heart from locally-based motion. Like the Geometry glyph, this glyph displays motion-based data, but provides further information on the motion by inferring physical properties.

The two primary facets of the glyph are the encoding of the strain magnitude \& direction, and the velocity magnitude \& direction. The strain magnitude is obtained from the first Eigenvalue of the strain tensor, and the strain direction is obtained from the first Eigenvector (the primary principle strain amount / direction). The velocity vector is sampled directly from the computed velocity field, and its length taken as the magnitude. For each time step in the tag tracking data, we have a series of vector pairs $\left(\left(s_{1}, v_{1}\right),\left(s_{2}, v_{2}\right), \ldots,\left(s_{n}, v_{n}\right)\right)$ to visually encode. Since direction is an important attribute, we choose direction as the predominant visual attribute and use the glyph's circumferential axis to show the vector directions and radial axis for the vector magnitudes. This encoding scheme implies no fixed 'time' axis, with the passing of time encoded by smoothly graduating the vector color.

\subsection{User Interface}

We developed a prototype system as shown in Fig. 3. The user can select different attribute fields available for visualization in different layers (Table 1). The user interaction for pinnable glyphs is particularly well designed to allow the users to place the glyph's probe inside the heart, but display the glyph outside the circular area which encloses the heart where minimal amount of occlusion is desirable. 


\section{Conclusions and Remaining Opportunities}

In this paper, we have considered the challenges and opportunities in CMR data visualization. While we have identified the relatively sparse research effort in this area, we have related the subject to several subareas of visualization, including time-varying volume visualization, video visualization, multi-field visualization and flow visualization. This suggests that while CMR data visualization is technically challenging, it can draw inspiration and techniques from the advances in other areas of visualization.

We have presented a case study on visualizing multi-field axial image sequences, which demonstrates the feasibility of integrating different visualization techniques into a common framework. Using this system we can begin to look back at how our system addresses some of the challenges outlined in Section 3. Our approach is to decompose the grand challenge of visualizing 4D scalar and vector fields into different user tasks, addressing each by using the most appropriate visualization techniques. Using a task-based approach with many visual designs allows us to address the task sensitivity challenge, looking towards a future with the possibility of using a unified system for the multiple objectives of clinical work, medical research, and doctor-patient communication. Our system does not rely upon image registration or segmentation methods, instead utilising user expertise for the placement of augmented visualization tools that we call pinnable glyphs. This manual approach reduces the requirement for high data quality which cannot be reasonably assumed in a clinical setting. These glyphs go some way towards addressing the dimensionality challenge by summarising temporal features using the available visual channels of the glyphs in a static manner. These glyphs are presented in a familiar context, i.e., animated CMR imagery, reducing the learning curve of such a system.

It is clear that there are many further opportunities for CMR visualization. In particular, we note the relative lack of research in clinical applications of visualization in CMR verses the large body of work for research settings. Perhaps it is timely to place CMR data visualization at the top of the research agenda in medical visualization.

[1] K. A. A. E. Waterfall, T. J. Atherton. 4D volume rendering with the shear warp factorisation. In Proc. IEEE Symposium on Volume Visualization, pages 129-137. 2000 .

[2] J. Blaas, C. P. Botha, and F. H. Post. Extensions of parallel coordinates for interactive exploration of large multi-timepoint data sets. IEEE Transactions on Visualization and Computer Graphics, 14(6):1436-1451, 2008.

[3] R. Borgo, M. Chen, B. Daubney, E. Grundy, H. Jänicke, G. Heidemann, B. Höferlin, M. Höferlin, D. Weiskopf, and X. Xie. State of the art report on video-based graphics and video visualization. Computer Graphics Forum, 31(8):2450-2477, 2012.

[4] R. Borgo, J. Kehrer, D. H. S. Chung, E. Maguire, R. S. Laramee, H. Hauser, M. Ward, and M. Chen. Glyph-based visualization: Foundations, design guidelines, techniques and applications. In Proc. Eurographics State-of-the-Art Reports. 2013. 
[5] R. P. Botchen, S. Bachthaler, F. Schick, G. M. M. Chen, D. Weiskopf, and T. Ertl. Action-based multifield video visualization. IEEE Transactions on Visualization and Computer Graphics, 14(4):885-899, 2008.

[6] T. Brox, A. Bruhn, N. Papenberg, and J. Weickert. High accuracy optical flow estimation based on a theory for warping. In Proc. ECCV, pages 25-36, 2004.

[7] J. Campbell and M. Shin. Essentials of Geographic Information Systems. Flatworld Knowledge, 2011.

[8] C.-K. Chen, C. Wang, K.-L. Ma, and A. T. Wittenberg. Static correlation visualization for large time-varying volume data. In Proc. IEEE Pacific Visualization, pages 27-34. 2011.

[9] M. Chen, R. Botchen, R. Hashim, D. Weiskopf, T. Ertl, and I. Thornton. Visual signatures in video visualization. IEEE Transactions on Visualization and Computer Graphics, 12(5):1093-1100, 2006.

[10] M. Chen and L. Floridi. An analysis of information visualisation. Synthese, 190(16):3421-3438, 2013.

[11] I. Codreanu, M. D. Robson, S. J. Golding, B. A. Jung, K. Clarke, and C. J. Holloway. Longitudinally and circumferentially directed movements of the left ventricle studied by cardiovascular magnetic resonance phase contrast velocity mapping. Journal of Cardiovascular Magnetic Resonance, 12:48, 2010.

[12] C. D. Correa and K.-L. Ma. Dynamic video narratives. ACM Transactions on Graphics, 29(3):88, 2010.

[13] G. Daniel and M. Chen. Video visualization. In Proc. IEEE Visualization, pages 409-416, 2003.

[14] P. Dragicevic, G. Ramos, J. Bibliowitcz, D. Nowrouzezahrai, R. Balakrishnan, and K. Singh. Video browsing by direct manipulation. In Proc. ACM CHI, pages 237-246, 2008.

[15] B. Duffy, J. Thiyagalingam, S. Walton, D. J. Smith, A. Trefethen, J. C. KirkmanBrown, E. A. Gaffney, and M. Chen. Glyph-based video visualization for semen analysis. IEEE Transactions on Visualization and Computer Graphics, to appear, 2014.

[16] M. Edmunds, R. S. Laramee, G. Chen, N. Max, E. Zhang, and C. Ware. Surfacebased flow visualization. Computers \& Graphics, 36(8):974 - 990, 2012. Graphics Interaction Virtual Environments and Applications 2012.

[17] A. Frydrychowicz, T. A. Bley, S. Dittrich, J. Hennig, M. Langer, , and M. Markl. Visualization of vascular hemodynamics in a case of a large patent ductus arteriosus using flow sensitive 3D CMR at 3T. Journal of Cardiovascular Magnetic Resonance, 9(3):585-587, 2007. 
[18] R. Fuchs and H. Hauser. Visualization of multi-variate scientific data. Computer Graphics Forum, 28(6):1670-1690, 2009.

[19] J. Gao, H.-W. Shen, J. Huang, and J. A. Kohl. Visibility culling for time-varying volume rendering using temporal occlusion coherence. In Proc. IEEE Visualization, pages 147-154. 2004.

[20] D. B. Goldman, B. Curless, S. M. Seitz, and D. Salesin. Schematic storyboarding for video visualization and editing. ACM Transactions on Graphics, 25(3):862871, 2006.

[21] M. A. Guttman, E. A. Zerhouni, and E. R. McVeigh. Analysis of cardiac function from MR images. IEEE Computer Graphics and Applications, 17(1):30-38, 1997.

[22] M. A. Guttman, E. A. Zerhouni, E. R. McVeigh, and M. A. Guttman. Analysis and visualization of cardiac function from mr images, 1997.

[23] M. Höferlin, E. Grundy, R. Borgo, D. Weiskopf, M. Chen, I. W. Griffiths, and W. Griffiths. Video visualization for snooker skill training. Computer Graphics Forum, 29(3):1053-1062, 2010.

[24] H.-W. S. J. Woodring. Multi-variate, time varying, and comparative visualization with contextual cues. IEEE Transactions on Visualization and Computer Graphics, 12(5):909-916, 2006.

[25] Y. Jang, D. Ebert, and K. Gaither. Time-varying data visualization using functional representations. IEEE Transactions on Visualization and Computer Graphics, Pre-publication:DOI 10.1109/TVCG.2011.54, 2011.

[26] A. Joshi and P. Rheingans. Illustration-inspired techniques for visualizing timevarying data. In Proc. IEEE Visualization, pages 679 - 686. 2005.

[27] H. Kang, X. Chen, Y. Matsushita, and X. Tang. Space-time video montage. In Proc. IEEE Computer Vision and Pattern Recognition, volume 2, pages 13311338, 2006.

[28] G. Kindlmann and C.-F. Westin. Diffusion tensor visualization with glyph packing. IEEE Transactions on Visualization and Computer Graphics, 12(5):13291336, 2006.

[29] R. M. Kirby, H. Marmanis, and D. H. Laidlaw. Visualizing multivalued data from $2 \mathrm{~d}$ incompressible flows using concepts from painting. In Proc. IEEE Visualization, pages 333-340, 1999.

[30] G. Klajnsek and B. Csebfalvi. 4D volume rendering by differential splatting. In Proc. 25th International Conference on Information Technology Interfaces, pages 651-656. 2003. 
[31] D. H. Laidlaw, E. T. Ahrens, D. Kremers, M. J. Avalos, R. E. Jacobs, and C. Readhead. Visualizing diffusion tensor images of the mouse spinal cord. In Proc. IEEE Visualization, pages 127-134, 1998.

[32] R. S. Laramee, H. Hauser, H. Doleisch, B. Vrolijk, F. H. Post, and D. Weiskopf. The state of the art in flow visualization: Dense and texture-based techniques. Computer Graphics Forum, 23:2004, 2004.

[33] T. Y. Lee and H.-W. Shen. Visualization and exploration of temporal trend relationships in multivariate time-varying data. IEEE Transactions on Visualization and Computer Graphics, 15(6):1359-1366, 2009.

[34] P. Legg, D. Chung, M. Parry, M. Jones, R. Long, I. Griffiths, and M. Chen. Matchpad: Interactive glyph-based visualization for real-time sports performance analysis. Computer Graphics Forum, 31(3):1255-1264, 2012.

[35] F. Leyva. Cardiac resynchronization therapy guided by cardiovascular magnetic resonance. Journal of Cardiovascular Magnetic Resonance, 12:64, 2010.

[36] F. Li, A. Gupta, E. Sanocki, L. He, and Y. Rui. Browsing digital video. In Proc. ACM CHI, pages 169-176, 2000.

[37] A. Lu and H.-W. Shen. Interactive storyboard for overall time-varying data visualization. In Proc. IEEE Pacific Visualization, pages 143-150. 2008.

[38] A. M. MacEachren. How Maps Work: Representation, Visualization and Design. Guilford Press, 2004.

[39] A. Mack and I. Rock. Inattentional Blindness. MIT Press, Cambridge, MA, 1998.

[40] E. Maguire, P. Rocca-Serra, S.-A. Sansone, J. Davies, and M. Chen. Taxonomybased glyph design - with a case study on visualizing workflows of biological experiments. IEEE Transactions on Visualization and Computer Graphics, 18(12):2603-2612, 2012.

[41] T. McLoughlin, R. S. Laramee, R. Peikert, F. H. Post, and M. Chen. Over Two Decades of Integration-Based, Geometric Flow Visualization. Computer Graphics Forum, 29(6):1807-1829, 2010.

[42] G. A. Miller. The magical number seven, plus or minus two: Some limits on our capacity for processing information. Psychological Review, 101(2):343?52, 1956.

[43] N. Neophytou and K. Mueller. Space-time points: 4D splatting on efficient grids. In Proc. IEEE/ACM SIGGRAPH Symposium on Volume Visualization and Graphics, pages 97-106. 2002.

[44] M. Nienhaus and J. Döllner. Depicting dynamics using principles of visual art and narrations. IEEE Computer Graphics and Applications, 25(3):40-51, 2005. 
[45] N. F. Osman and J. L. Prince. Visualizing myocardial function using harp mri. Physics in Medicine and Biology, 45(6):1665, 2000.

[46] N. Patel and I. Sethi. Video shot detection and characterization for video databases. Pattern Recognition, 30(4):538-592, 1997.

[47] G. M. Pohost. The history of cardiovascular magnetic resonance. JACC Cardiovascular and Imaging, 1:672-678, 2008.

[48] M. Poon, V. Fuster, and Z. Fayad. Curr Opin Cardiol, 17:663-70, 2002.

[49] F. H. Post, B. Vrolijk, H. Hauser, R. S. Laramee, and H. Doleisch. The state of the art in flow visualisation: Feature extraction and tracking, 2003.

[50] A. N. Price, K. K. Cheung, J. O. Cleary, A. E. Campbell, J. Riegler, and M. F. Lythgoe. Cardiovascular magnetic resonance imaging in experimental models. The Open Cardiovascular Medicine Journal, 4:278-292, 2010.

[51] R. Ramos, G.and Balakrishnan. Fluid interaction techniques for the control and annotation of digital video. In Proc. ACM Symposium on User Interface Software and Technology, pages 105-114, 2003.

[52] T. Ropinski, S. Oeltze, and B. Preim. Survey of Glyph-based Visualization Techniques for Spatial Multivariate Medical Data. Computers \& Graphics, 2011.

[53] T. Ropinski and B. Preim. Taxonomy and usage guidelines for glyph-based medical visualization. In Proceedings of the 19th Conference on Simulation and Visualization (SimVis08), pages 121-138, 2008.

[54] K. Schoeffmann and L. Boeszoermeny. Video browsing using interactive navigation summaries. In Proc. International Workshop on Content-Based Multimedia Indexing, pages 243-248, 2009.

[55] H.-W. Shen, L.-J. Chiang, and K.-L. Ma. A fast volume rendering algorithm for time-varying fields using a time-space partitioning (tsp) tree. In Proc. IEEE Visualization, pages 371-377. 1999.

[56] D. Silver and X. Wang. Tracking and visualizing turbulent 3D features. IEEE Transactions on Visualization and Computer Graphics, 3(2):129-141, 1997.

[57] D. Simons and R. Rensink. Change blindness: Past, present, and future. Trends in Cognitive Sciences, 9(1):16-20, 2005.

[58] P. M. Sutton and C. D. Hansen. Isosurface extraction in time-varying fields using a temporal branch-on-need tree(t-bon). In Proc. IEEE Visualization, pages 147154. 1999.

[59] N. A. Svakhine, Y. Jang, D. Ebert, and K. Gaither. Illustration and photography inspired visualization of flows and volumes. In Proc. IEEE Visualization, pages 687-694. 2005. 
[60] R. Taylor. Visualizing multiple fields on the same surface. IEEE Computer Graphics and Applications, 22(3):6-10, 2002.

[61] J. Töger, M. Carlsson, G. Söderlind, H. Arheden, and E. Heiberg. Volume tracking: A new method for quantitative assessment and visualization of intracardiac blood flow from three-dimensional, timeresolved, three-component magnetic resonance velocity mapping. BMC Medical Imaging, 11:10, 2011.

[62] V. S. Truong B. Video abstraction: A systematic review and classification. ACM Transactions on Multimedia Computing, Communications and Applications, 3(1), 2007.

[63] R. F. P. van Pelt, S. S. A. M. Jacobs, B. M. ter Haar Romenij, and A. Vilanova. Visualization of 4D blood-flow fields by spatiotemporal hierarchical clustering. Computer Graphics Forum, 31(3):1065-1074, 2012.

[64] R. F. P. van Pelt, J. Oliván Bescós, M. Breeuwer, R. E. Clough, M. E. Gröller, B. M. ter Haar Romeny, and A. Vilanova. Interactive virtual probing of 4D MRI blood-flow. IEEE Transactions on Visualization and Computer Graphics, 17(12):2153-2162, 2011.

[65] C. Wang, J. Gao, L. Li, and H.-W. Shen. A multiresolution volume rendering framework for large-scale time-varying data visualization. In Proc. Volume Graphics, pages 11-19. 2005.

[66] M. Ward. Multivariate data glyphs: Principles and practice. In C.-H. Chen, W. K. Härdle, and A. Unwin, editors, Handbook of Data Visualization, page 179?198. Springer Handbooks Computational Statistics, 2008.

[67] J. Woodring, C. Wang, and H.-W. Shen. High dimensional direct rendering of time-varying volumetric data. In Proc. IEEE Visualization, pages 417-424. 2003.

[68] B. C. Wünsche, R. Lobb, and A. A. Young. The visualization of myocardial strain for the improved analysis of cardiac mechanics. In Proc. GRAPHITE, pages 9099, 2004.

[69] C. Zhang, D. Xue, R. Crawfis, and R. Wenger. Time-varying interval volumes. In Proc. Volume Graphics, pages 99-108. 2005. 